\title{
Levantamento dos agravos de notificação compulsória dos profissionais da saúde do
}

\section{Rio Grande do Norte}

\author{
Survey of compulsory notification diseases of health professionals in Rio Grande do Norte \\ Levantamiento de los agravios de notificación obligatoria de los profesionales de la salud de Rio
}

Grande do Norte

Recebido: 23/04/2021 | Revisado: 03/05/2021 | Aceito: 06/05/2021 | Publicado: 21/05/2021

\author{
Anayla Oliveira da Silva \\ ORCID: https://orcid.org/0000-0002-8112-1650 \\ Universidade Potiguar, Brasil \\ E-mail: anaylasilva@hotmail.com \\ Luana Araújo dos Santos \\ ORCID: https://orcid.org/0000-0003-1849-4531 \\ Universidade Potiguar, Brasil \\ E-mail: luhanaraujo@gmail.com \\ Wittally Lima de Araújo \\ ORCID: https://orcid.org/0000-0002-5620-0023 \\ Universidade Potiguar, Brasil \\ E-mail: wittally@hotmail.com \\ Cleuton Braz Morais \\ ORCID: https://orcid.org/0000-0002-7917-3363 \\ Universidade Estadual do Rio Grande do Norte, Brasil \\ E-mail: cleutonbraz@icloud.com \\ Nathane Marluce Vieira de Araújo \\ ORCID: https://orcid.org/0000-0002-0915-6971 \\ Universidade Potiguar, Brasil \\ E-mail: nathane_vieira@hotmail.com \\ Mirlucia Ferreira da Rocha Silva \\ ORCID: https://orcid.org/0000-0001-9313-9826 \\ Universidade Potiguar, Brasil \\ E-mail: mirluciarocha@gmail.com \\ Francisco de Assis de Souza Júnior \\ ORCID: https://orcid.org/0000-0003-3834-2690 \\ Universidade Potiguar, Brasil \\ E-mail: souzajuniorfa@hotmail.com
}

\begin{abstract}
Resumo
A saúde do trabalhador representa uma área da saúde pública que visa analisar e intervir sobre as relações de trabalho que resultem em doenças e agravos contribuindo para a melhoria da qualidade de vida. Analisar a prevalência de profissionais da saúde que sofreram agravos relacionados ao trabalho no estado do Rio Grande do Norte. Pesquisa transversal de prevalência, que teve como critérios de inclusão agravos/doenças a saúde do trabalhador nas seguintes categorias profissionais: Médico, Cirurgião-Dentista, Enfermeiro, Técnico de enfermagem e Auxiliar de Saúde Bucal. Como critério de exclusão, não foram incluídas informações que comprometiam a integridade pessoal das vítimas de acidente de trabalho. Como resultado foi apontado que o técnico de enfermagem é responsável pela maioria dos números de casos em todos os agravos relacionados ao trabalho notificados no estado do Rio Grande do Norte. O agravo mais notificado nesta pesquisa, entre 2008 e 2018, foi a exposição a material biológico, com $98 \%$, seguido pelo acidente de trabalho grave, com $1 \%$ e transtorno mental, com $1 \%$. Foi constatado também que as frequências das notificações vêm aumentando ao longo dos anos. Estes resultados evidenciam necessidade de maior consciência do processo de trabalho e realização de atividades preventivas, visto que a saúde do trabalhador é um eixo importante da saúde pública. Conclui-se que há uma significativa prevalência de acidentes ocupacionais os quais envolvem os profissionais da saúde, evidenciando que existe uma falha na execução de suas funções, possivelmente por não utilizar protocolos simples de prevenção. Por isso, recomenda-se que os profissionais da saúde do Rio Grande do Norte ampliem a consciência do seu processo de trabalho, adquirindo conhecimento científico para prevenção dos agravos à saúde.
\end{abstract}

Palavras-chave: Agravos; Saúde do trabalhador; Epidemiologia. 


\begin{abstract}
The worker's health is an area of public health that aims to analyze and intervene on work relations that result in diseases and diseases contributing to the improvement of the quality of life. To analyze the prevalence of health professionals who suffered work-related injuries in the state of Rio Grande do Norte. Methodology: Cross-sectional study of prevalence, which had as inclusion criteria diseases/occupational health in the following professional categories: Physician, Dental Surgeon, Nurse, Nursing Technician and Oral Health Assistant. As an exclusion criterion, no information was included that would compromise the personal integrity of victims of accidents at work. Results and Discussion: As a result, it was pointed out that the nursing technician is responsible for the majority of cases in all work-related diseases reported in the state of Rio Grande do Norte. The most reported problem in this research, between 2008 and 2018, was exposure to biological material, with $98 \%$, followed by serious work accident, with $1 \%$ and mental disorder, with $1 \%$. It was also found that the frequency of notifications has been increasing over the years. These results show the need for greater awareness of the work process and preventive activities, since the worker's health is an important axis of public health. Conclusions: It is concluded that there is a significant prevalence of occupational accidents involving health professionals, evidencing that there is a failure in the performance of their functions, possibly because they do not use simple prevention protocols. Therefore, it is recommended that health professionals in Rio Grande do Norte expand their awareness of their work process, acquiring scientific knowledge to prevent health problems.
\end{abstract}

Keywords: Health problems; Occupational health; Epidemiology.

\title{
Resumen
}

La salud del trabajador representa un área de la salud pública que busca analizar e intervenir sobre las relaciones de trabajo que resulten en enfermedades y agravios contribuyendo para la mejora de la calidad de vida. Analizar la prevalencia de profesionales de la salud que sufrieron agravios relacionados con el trabajo en el estado de Rio Grande do Norte. Metodología: Investigación transversal de prevalencia, que tuvo como criterios de inclusión agravios/enfermedades la salud del trabajador en las siguientes categorías profesionales: Médico, Cirujano-Dentista, Enfermero, Técnico de enfermería y Auxiliar de Salud Bucal. Como criterio de exclusión, no se incluyó información que comprometiera la integridad personal de las víctimas de accidentes laborales. Resultados y Discusión: Como resultado se señaló que el técnico de enfermería es responsable de la mayoría de los números de casos en todos los agravios relacionados con el trabajo notificados en el estado de Rio Grande do Norte. El agravio más notificado en esta investigación, entre 2008 y 2018, fue la exposición a material biológico, con 98\%, seguido por el accidente de trabajo grave, con $1 \%$ y trastorno mental, con $1 \%$. También se ha constatado que las frecuencias de las notificaciones han ido aumentando a lo largo de los años. Estos resultados evidencian necesidad de mayor conciencia del proceso de trabajo y realización de actividades preventivas, ya que la salud del trabajador es un eje importante de la salud pública. Conclusiones: Se concluye que hay una significativa prevalencia de accidentes ocupacionales en los que participan los profesionales de la salud, evidenciando que existe una falla en la ejecución de sus funciones, posiblemente por no utilizar protocolos simples de prevención. Por eso, se recomienda que los profesionales de la salud de Rio Grande do Norte amplíen la conciencia de su proceso de trabajo, adquiriendo conocimiento científico para la prevención de los agravios a la salud.

Palabras clave: Agravios; Salud del trabajador; Epidemiología.

\section{Introdução}

Nos anos 60 ocorreram diversos avanços científicos importantes na medicina e na saúde pública ampliando os saberes sobre o processo saúde-doença e sua interferência com o trabalho. A saúde do trabalhador é um direito universal, sendo uma resposta aos anseios dos movimentos sociais (Gomez \& Vasconcellos, 2018).

Os primeiros programas e nos Centros de Referências em Saúde do Trabalhador - CRST, prevalecia o âmbito assistencial, com finalidade e função de diagnosticar, orientar e acompanhar as patologias que poderiam surgir decorrentes do trabalho criando condições para que as redes públicas desenvolvessem métodos efetivos para a assistência à saúde dos trabalhadores (Gomez \&Vasconcellos, 2018).

De acordo com a Organização Mundial da Saúde, os maiores desafios enfrentados pelos trabalhadores são os problemas de saúde ocupacional ligados com as novas tecnologias de informação e automação, novas substâncias químicas, riscos de saúde associados a novas biotecnologias, envelhecimento da população trabalhadora, problemas relacionados com a crescente mobilidades dos trabalhadores e ocorrência de novas doenças ocupacionais de várias origens (OPAS/OMS, 2017).

A Política Nacional de Saúde do Trabalhador do Ministério da Saúde instituída em 2004 visa à redução dos acidentes e doenças relacionadas ao trabalho, mediante a execução de ações de promoção, reabilitação e vigilância na área de saúde. 
No Brasil, o acidente de trabalho é considerado um agravo de notificação compulsória contemplando o acidente grave, fatal, em crianças e adolescentes e com exposição à material biológico. Agravo é qualquer dano à integridade física ou mental do indivíduo, provocado por circunstâncias nocivas, tais como acidentes, intoxicações por substâncias químicas, abuso de drogas ou lesões decorrentes de violências interpessoais, como agressões e maus tratos, e lesão autoprovocada (Brasil, 2020).

De acordo com a nota informativa $n^{\circ} 94$ de 2019 do Ministério da Saúde, acidentes de trabalho podem ocorrer no ambiente de trabalho ou durante o exercício do trabalho externo provocando lesão corporal ou perturbação funcional, podendo causar a perda ou redução temporária ou permanente da capacidade para o trabalho ou morte. As doenças ocupacionais representam importante parcela da carga de enfermidade ao redor do mundo, atingindo 2,4 milhões de pessoas/ano (Hämäläinen, Takala \& Kiat, 2017).

A Portaria 204, de 17 de fevereiro de 2016, revogada pela Portaria de Consolidação n ${ }^{\circ} 04$ de 28 de setembro de 2017, os acidentes de trabalho grave e acidentes de trabalho com exposição a material biológico foram definidos como agravos de notificação compulsória universal.

Com a publicação da Portaria 205, de 17 de fevereiro de 2016, revogada pela Portaria de Consolidação ${ }^{\circ}$ 5, de 28 de setembro de 2017, as doenças e agravos de notificação compulsória são: Acidente Grave, Câncer relacionado ao trabalho Dermatoses ocupacionais, Lesão por Esforço Repetitivo/Distúrbio Osteomuscular Relacionado ao Trabalho (LER/DORT), Perda Auditiva Induzida por Ruído (PAIR) relacionada ao trabalho, Pneumoconioses relacionadas ao trabalho e Transtornos mentais relacionados ao trabalho (Brasil, 2017).

O acidente por material biológico se dar pela exposição direta ou indireta do trabalhador por patógenos (vírus, bactérias, fungos, príons e protozoários) e/ou por meio de material perfurocortante ou não. Em relação aos casos de câncer desenvolvidos devido ao trabalho, a exposição a fatores, agentes e situações de risco presentes no ambiente e o processo de trabalho, mesmo após cessada a exposição, são considerados causas para o seu desenvolvimento. Problemas de saúde envolvendo dermatoses, doenças pulmonares e diminuição da audição também são relatadas, além dos casos das lesões e síndromes que afetam o sistema músculo esquelético, causadas ou agravadas pelo trabalho, como no caso das LER/DORT (Brasil, 2019).

Os profissionais de saúde estão o tempo todo acompanhando pessoas em processos de adoecimento, perdas e luto por meio das relações diárias. De forma pessoal, também estão vivenciando suas próprias perdas e consequentes lutos. Por isso, precisam buscar vias de expressão emocional e suporte para que possam lidar com diversas situações de sofrimento psicológico, vivenciadas em seu ambiente de trabalho e/ou domiciliar (EBSERH, 2018). Na categoria de transtornos mentais relacionados ao trabalho, a nota informativa de $\mathrm{n}^{\circ} 94$ de 2019 do ministério da saúde evidencia todo caso de sofrimento emocional que podem indicar o desenvolvimento ou agravo de transtornos mentais, além da síndrome de Burnout, sintomas e sinais relativos ao estado emocional, comportamento e lesão autoprovocada intencionalmente (Brasil, 2019).

Com isso, a Vigilância em Saúde do Trabalhador (Visat) tem por objetivo a proteção e promoção à saúde dos trabalhadores por meio de atenção integral, desenvolvendo ações junto com a principal estratégia do Ministério da Saúde a Rede Nacional de Atenção Integral à Saúde do Trabalhador (RENAST), implementado em 2002 por meio da Portaria $\mathrm{n}^{\circ}$ 1.679/GM, com o objetivo de efetivar ações de promoção, prevenção, assistência e vigilância em Saúde do Trabalhador em todos os serviços do SUS, tendo como ordenadora da rede, a atenção básica, e os Centros de Referência em Saúde do Trabalhador (CEREST) (Cavalcante, Oliveira Costa, Medeiros \& Menezes, 2015).

Em 2012, a RENAST passou a ser a principal estratégia da organização da Saúde do Trabalhador no SUS. O CEREST tornou-se nos âmbitos regionais e estaduais, o principal responsável pela implementação e fortalecimento da Vigilância em Saúde do Trabalhador os quais visam melhorias das condições de trabalho e na qualidade de vida dos trabalhadores 
(Brasil,2017).

O CEREST é responsável por agregar as notificações de acidentes de trabalho graves e daqueles com exposição a material biológico, no Sistema de Informações de Agravos de Notificação (SINAN), que possui como objetivo a coleta, transmissão e disseminação dos dados gerados rotineiramente pelo Sistema de Vigilância Epidemiológica das três esferas de governo, através de uma rede informatizada, com o intuito de apoiar o processo de investigação e dar subsídios à análise das informações de vigilância epidemiológica dos agravos de notificações compulsórias (Possebom \& Alonço, 2018).

O Sinan é alimentado, principalmente, pela notificação e investigação de casos de doenças e agravos que constam da lista nacional de doenças de notificação compulsória, permitindo a realização do diagnóstico dinâmico da ocorrência de um evento na população, podendo fornecer subsídios para explicações causais dos agravos de notificação compulsória, além de vir a indicar riscos aos quais as pessoas estão sujeitas, contribuindo assim, para a identificação da realidade epidemiológica de determinada área geográfica. O seu uso sistemático, de forma descentralizada, contribui para a democratização da informação, permitindo que todos os profissionais de saúde tenham acesso à informação e as tornem disponíveis para a comunidade. É, portanto, um instrumento relevante para auxiliar o planejamento da saúde, definir prioridades de intervenção, além de permitir que seja avaliado o impacto das intervenções (Brasil, 2016).

Nos últimos anos, a saúde do trabalhador tem conquistado maior espaço nas pautas de discussões que envolvem relações de trabalho. Além disso, houve o reconhecimento, por parte dos empregadores, da necessidade de investimento nessa área. Dados do Anuário Estatístico de Acidentes de Trabalho (AEAT) mostram que, em 2013, a Previdência Social concedeu 338,1 mil benefícios acidentários (Secretaria de previdência, 2020).

A importância e relevância dessa pesquisa se dá pelo fato de os profissionais da saúde estarem expostos a agravos relacionados ao trabalho pois atuam rotineiramente prestando assistência ao paciente através do contato direto e apesar do conhecimento sobre as possíveis consequências de acidentes ocupacionais é estipulado que na prática eles subestimam os riscos, levando a negligência das normas de biossegurança. Mediante ao exposto, faz-se necessário um estudo que proporcione avaliar quantitativamente os profissionais da saúde que sofreram agravos relacionados ao trabalho no estado do Rio Grande do Norte. Pois é de fundamental importância colaborar com a saúde pública do estado visando sempre o aprimoramento e discussão acerca dessa temática.

O objetivo deste trabalho é avaliar os agravos ocorridos durante as atividades laborativas dos profissionais de saúde do Rio Grande do Norte, relacionando a prevalência de casos de notificação compulsória no Sistema de Informação de Agravos de Notificações (SINAN).

\section{Metodologia}

Estudo transversal de prevalência, realizado na Secretaria de Estado da Saúde Pública do Rio Grande do Norte (SESAP-RN) localizada na Avenida Deodoro da Fonseca, 730, Cidade Alta, Natal - RN, CEP: 59025-600. Submetido à apreciação pelo comitê de ética em pesquisa da Liga Norte Riograndense Contra o Câncer com parecer favorável, sem restrições (CAAE: 16024419.9.0000.5296).

Os Critérios de inclusão foram relacionados aos agravos/doenças que afetam a saúde do trabalhador das categorias profissionais: Médico, Cirurgião-Dentista, Enfermeiro, Técnico de enfermagem e Auxiliar de Saúde Bucal. Recolhendo notificações entre o período de 2008 a 2018 no estado do Rio Grande do Norte nos seguintes eixos: acidente de trabalho grave; câncer relacionado ao trabalho; dermatoses ocupacionais; exposição a material biológico; lesão por esforço repetitivo/doenças osteomusculares relacionadas ao trabalho; a perda auditiva induzida por ruído; pneumoconiose e transtorno mental.

No agravo de acidentes com exposição a material biológico, foi incluído: circunstância do acidente e a evolução do caso, além de profissionais de todas as idades e localidades do estado do Rio Grande do Norte. Como critério de exclusão, não 
foram avaliadas informações que comprometiam a integridade pessoal das vítimas de acidente de trabalho.

Para a obtenção dos dados, foi utilizada as informações coletadas da Ficha Individual de Notificação (FIN) que são preenchidas pelas unidades assistenciais para cada paciente quando da suspeita da ocorrência de problema de saúde de notificação compulsória de interesse nacional, estadual ou municipal. Esses dados são repassados semanalmente para as Secretarias Estaduais de Saúde (SES). Os dados foram analisados através de estatística descritiva, mediante cálculo de média simples com valores absolutos e porcentagens e mostrados a partir de tabelas. Esse artigo é parte integrante da pesquisa "Levantamento dos agravos de notificação compulsória dos profissionais da saúde do Rio Grande do Norte", que teve como benefício identificar as categorias profissionais e os agravos mais comuns no ambiente de trabalho, realizando uma discussão acerca dos cuidados e medidas que devem ser tomadas para evitar esses acidentes.

\section{Resultados}

De acordo com o levantamento dos dados durante a pesquisa, os agravos relacionados ao trabalho; como o câncer, perda auditiva induzida por ruído e pneumoconiose, não foram notificados dentro das categorias profissionais analisadas neste estudo, durante o período de 2008 a 2018 no estado do Rio Grande do Norte. A tabela 1 mostra todos os dados levantados durante a pesquisa, evidenciando bem quais categorias mais sofrem com os agravos de saúde decorrentes da sua profissão.

Tabela 1 - Total de casos notificados de agravos relacionados ao trabalho, segundo a ocupação no estado do Rio Grande do Norte de 2008 a 2018.

\begin{tabular}{ccc} 
OCUPAÇÕES & NOTIFICAÇÕES & $\%$ \\
\hline Médico & 511 & $8 \%$ \\
\hline Cirurgião-Dentista & 342 & $5 \%$ \\
\hline Enfermeiro & 727 & $12 \%$ \\
\hline Técnico de enfermagem & 4.590 & $73 \%$ \\
\hline ASB & 126 & $2 \%$ \\
\hline TOTAL & $\mathbf{6 . 2 9 6}$ & $\mathbf{1 0 0}$
\end{tabular}

Fonte: Sistema de Informação de Agravos de Notificação (2008 a 2018).

Dos 6.296 casos notificados, $73 \%$ evidenciaram o técnico de enfermagem como a categoria profissional mais afetada pelos agravos relacionados a ocupação no período de 2008 a 2018 no estado do Rio Grande do Norte.

A avaliação do total de casos de acidente de trabalho grave notificados, segundo a ocupação, foi de $75 \%$ para técnicos de enfermagem seguido de $20 \%$ para enfermeiros, sendo o restante das ocupações com pouca relevância nessa notificação. Nos casos de dermatoses ocupacionais notificados, segundo a ocupação, mais uma vez os técnicos de enfermagem e os enfermeiros foram os profissionais mais notificados, somando 100\% dos casos no período de 2008 a 2018.

O levantamento dos casos de exposição a material biológico foi analisado de acordo com a categoria profissional e o número de notificações decorrentes no período, obtendo os dados da tabela abaixo. 
Tabela 2 - Total de casos de exposição a material biológico notificados, segundo a ocupação, estado do Rio Grande do Norte, 2008 a 2018.

\begin{tabular}{ccc} 
OCUPAÇÕES & NOTIFICAÇÕES & $\%$ \\
\hline Médico & 510 & $8 \%$ \\
\hline Cirurgião-Dentista & 340 & $6 \%$ \\
\hline Enfermeiro & 706 & $11 \%$ \\
\hline Técnico de enfermagem & 4.478 & $73 \%$ \\
\hline ASB & 123 & $2 \%$ \\
\hline TOTAL & $\mathbf{6 . 1 5 7}$ & $\mathbf{1 0 0}$
\end{tabular}

Fonte: Sistema de Informação de Agravos de Notificação (2008 a 2018).

A categoria profissional com maiores casos de exposição a material biológico, de acordo com o levantamento, são os técnicos de enfermagem, com 4.478 (73\%) dos casos notificados. Para as Lesão por Esforço Repetitivo/Doenças Osteomusculares Relacionadas ao Trabalho (LER/DORT), os casos de notificação foram poucos, levando em consideração o período de 10 anos analisado.

Tabela 3 - Total de casos de LER/DORT notificados, segundo a ocupação no estado do Rio Grande do Norte de 2008 a 2018.

\begin{tabular}{ccc} 
OCUPAÇÕES & NOTIFICAÇÕES & $\%$ \\
\hline Médico & 0 & $5 \%$ \\
\hline Cirurgião-Dentista & 1 & $5 \%$ \\
\hline Enfermeiro & 1 & $85 \%$ \\
\hline Técnico de enfermagem & 18 & $5 \%$ \\
\hline ASB & 1 & $\mathbf{1 0 0}$
\end{tabular}

Fonte: Sistema de Informação de Agravos de Notificação (2008 a 2018).

Com o levantamento desses dados, notou-se mais uma vez que o técnico de enfermagem é o profissional com maiores casos de notificação em relação aos agravos de saúde do trabalhador aqui relatados, contabilizando 18 notificações (85\%) no período de 2008 a 2018 avaliado. Para os transtornos mentais avaliados, apenas duas categorias de profissionais de saúde notificaram esse agravo durante o período de estudo, de a cordo com a tabela a seguir.

Tabela 4 - Total de casos de Transtorno Mental notificados, segundo a ocupação no Rio Grande do Norte de 2008 a 2018.

\begin{tabular}{ccc} 
OCUPAÇÕES & NOTIFICAÇÕES & $\%$ \\
\hline Médico & 0 & $0 \%$ \\
\hline Cirurgião-Dentista & 0 & $0 \%$ \\
\hline Enfermeiro & 3 & $91 \%$ \\
\hline Técnico de enfermagem & 31 & $0 \%$ \\
\hline ASB & 0 & $\mathbf{1 0 0}$
\end{tabular}

Fonte: Sistema de Informação de Agravos de Notificação (2008 a 2018). 
Das duas categorias notificadas em relação ao agravo do transtorno mental, o técnico de enfermagem aparece com 31 (91\%) dos casos relatados. Na tabela abaixo o total de casos de agravos relacionados ao trabalho nas cedes de cada Regionais Administrativas é distribuído da seguinte forma:

Tabela 5 - Total de casos nas regionais administrativas do Rio Grande do Norte de 2008 a 2018.

\begin{tabular}{ccc} 
REGIONAIS & NOTIFICAÇÕES & $\%$ \\
\hline Grande Natal & 4.740 & $75 \%$ \\
\hline I & 103 & $2 \%$ \\
\hline II & 956 & $15 \%$ \\
\hline III & 106 & $4 \%$ \\
\hline IV & 234 & $1 \%$ \\
\hline V & 102 & $1 \%$ \\
\hline VI & 55 & $\mathbf{1 0 0}$
\end{tabular}

A prevalência dos casos nas sete regionais administrativas do Rio Grande do Norte aponta para uma elevada proporção de trabalhadores, cerca de 75\%, notificados na Grande Natal.

O levantamento sobre o total de casos notificados de exposição à material biológico, de acordo com a ocupação, segundo a evolução do caso, se deu por 59\% não especificando a evolução, tendo registro como Branco/Ignorado.

\section{Discussão}

As atividades laborais desenvolvidas pelos profissionais de saúde os expõem a riscos físicos, químicos, biológicos, ergonômicos e psicossociais. Essa exposição contínua e múltipla potencializa a promoção do adoecimento dessa classe e promovem prejuízos às instituições governamentais e as organizações de saúde empregadoras, interferindo na qualidade da assistência ofertada aos usuários, pois o estado de saúde possui influência direta nas atividades executadas pelos trabalhadores. Diante disso, atualmente o acidente de trabalho é considerado um importante problema de saúde pública (Gomez, Vasconcellos \& Machado, 2018).

Segundo o Ministério da Saúde sobre as notificações relacionadas ao trabalho no período compreendido entre 2010 e 2015, temos o acidente de trabalho grave com 54,3\% de casos, seguidos pelos acidentes de trabalho com exposição a material biológico, com 34,2\% e lesão por esforço repetitivo/doenças osteomusculares relacionadas ao trabalho (LER/DORT), com $5,8 \%$ (Brasil, 2017).

O agravo mais notificado nesta pesquisa, entre 2008 e 2018, foi a exposição a material biológico, com 98\%, seguido pelo acidente de trabalho grave, com $1 \%$ e transtorno mental, com $1 \%$. Provavelmente, obtivemos estes resultados devido esse estudo analisar apenas as categorias profissionais que estão mais expostas ao contato direto com o paciente, evidenciando assim a elevada influência da exposição a material biológico a essas áreas específicas da saúde.

No estudo de Carvalho et al, concluíram que o profissional de Enfermagem apresenta maior percentual de acidentes, possivelmente, pela elevada frequência na exposição de tarefas que representam fatores de riscos para o acidente ocupacional. É a profissão com maior prevalência de desgaste físico e mental devido cobranças existentes e sobrecarga de trabalho, gerando estresse e a presença de outras enfermidades (Carvalho \& Luz, 2018). 
No entanto, o presente estudo apontou que o técnico de enfermagem é responsável pela maioria dos números de casos em todos os agravos relacionados ao trabalho notificados no estado do Rio Grande do Norte, tanto ao analisar o quantitativo total entre 2008 e 2018, como também ao realizar uma análise em cada agravo estudado. Esse resultado é alarmante, pois apesar do conhecimento ofertado pela literatura, em que essa categoria é amplamente reconhecida pela maior ocorrência de acidentes ocupacionais, atualmente as ações governamentais ainda são ineficazes quanto a conscientização dessa classe para se minimizar esses agravos no estado do Rio Grande do Norte.

Na pesquisa, é observado um alto percentual de respostas quanto a evolução do caso em branco/ignorado resultando no sub-registro, tornando-se necessário enfatizar medidas para o treinamento do preenchimento das fichas com o intuito de obter a melhor qualidade das informações.

É importante considerar que a subnotificação dos acidentes e das doenças relacionadas ao trabalho pode estar vinculada a diversos fatores, referentes tanto a trabalhadores quanto a empregadores. No que se refere à classe trabalhadora, $\mathrm{o}$ sub-registro pode estar relacionada ao desconhecimento dos sujeitos acerca de seus direitos legais ou pela não atribuição de importância à notificação do acidente. No que se refere aos empregadores e gestores, a subnotificação pode se dar tanto pelo desconhecimento como pelo descumprimento das legislações trabalhistas (Santana, Sarquis \& Miranda, 2020).

Apesar dos casos de subnotificação ainda serem bastante relevantes, o Ministério da Saúde, comparando-se a totalidade de notificações relacionadas ao trabalho entre 2010 e 2015, observou um aumento de 74,4\% nos registros. O que pode ter sido desencadeado devido as ações do RENAST (Rede Nacional de Atenção à Saúde do Trabalhador), da execução das metas do SISPACTO (Pactuação de Diretrizes, Objetivos, Metas e Indicadores), bem como, o envolvimento e conscientização dos profissionais das unidades notificadas (Brasil, 2017).

Em muitos casos, a empresa não reconhece que sua atividade tem sido colaboradora no desenvolvimento de agravos psíquicos pelos trabalhadores (Previdência social, 2017). O diretor do Departamento de Políticas de Saúde e Segurança Ocupacional do Ministério da Previdência, Marco Pérez, observou que apesar da resistência, ainda existente, houve um aumento nos esforços dos trabalhadores, empregadores e do governo, assim como das políticas intersetoriais de promoção de saúde e ambientes de trabalho mais seguros (Ministério da Previdência, 2020).

Com a Reforma Trabalhista, realizada no Brasil em 2017, potencializa a exposição de trabalhadores a riscos no ambiente de trabalho configurando-se como um retrocesso dos trabalhadores na área da saúde. $\mathrm{O}$ risco de adoecimento por conta do trabalho e as recentes mudanças na legislação trabalhista podem contribuir para o desgaste físico e emocional dos trabalhadores devido ao risco de sobrecarga de trabalho (Lei $\mathrm{N}^{\circ}$ 13.467, de 13 de julho de 2017).

Devido a isso, é necessário um esforço conjunto por parte das entidades de classe, dos trabalhadores e da sociedade para ampliar as discussões acerca dos efeitos nocivos dos riscos psicossociais, bem como a necessidade do desenvolvimento de medidas protetivas à saúde do trabalhador. É preciso reconhecer legalmente os riscos psicossociais como riscos ocupacionais no país e instituir programas de gerenciamento dos mesmos em todos os ambientes ocupacionais de modo a reduzir os impactos negativos na saúde e no bem-estar dos trabalhadores (Santana et al., 2020)

Cabe ao Ministério da Saúde e ao Ministério da Economia, no âmbito de suas respectivas competências promoverem maior transparência na disponibilização atualizada dos dados estatísticos relacionados a morbimortalidade entre os/as trabalhadores/as, bem como a ampla e adequada divulgação legal da notificação compulsória junto aos Centros de Referência em Saúde do Trabalhador (CEREST) e RENAST (Brasil, 2020).

A prevenção do acidente de trabalho e a capacitação e informação do trabalhador sobre a importância da segurança na execução de sua atividade laboral podem ser fortes aliadas. A maioria dos acidentes de trabalho poderiam ter sido evitados com o uso adequado dos EPIs (Possebom \& Alonço, 2018). Dentre as medidas de prevenção, destaca-se o uso dos EPIs na manutenção da segurança do trabalhador (Meireles \& Oliveira Pinto, 2016). 
Percebeu-se que durante a execução da pesquisa, a saúde do trabalhador é um eixo importante de saúde pública e a atuação do CEREST é essencial por dar subsídio técnico para o SUS na integralidade da assistência aos trabalhadores.

As dificuldades encontradas se deram a limitações na coleta de dados que em alguns casos, o sistema informatizado era incapaz de obter todas as variáveis, sendo necessário fragmentar as informações.

A população estudada nesta pesquisa possui uma representação significativa do estado do Rio Grande do Norte, tendo seus resultados e reflexões relevantes para o planejamento de intervenções de ações governamentais e por fornecer subsídios para pesquisas futuras.

\section{Conclusão}

É possível observar uma significativa prevalência de acidentes ocupacionais os quais envolvem os profissionais da saúde, evidenciando que existe uma falha na execução de suas funções, possivelmente por não utilizar protocolos simples de prevenção. A categoria mais afetada aos agravos relacionados ao trabalho foi o técnico de enfermagem, provavelmente por encontrar-se mais expostos a atividades que são consideradas fatores de riscos a esses agravos.

A exposição a material biológico é o agravo com mais notificações (98\% dos casos) e apesar dos índices alarmantes o seu tratamento ainda é negligenciado, resultando na difícil resolução do caso. A prevalência de casos de acordo com os levantamentos nas regionais administrativas do Rio Grande do Norte aponta para uma elevada proporção na Grande Natal, sendo pertinente destacar que essa região possui maior densidade demográfica quando comparada aos demais campos de estudo ou devido ocorrer uma maior procura desse público-alvo em busca de tratamento especializado.

Recomenda-se que os profissionais da saúde do Rio Grande do Norte incorporem ao seu cotidiano medidas preventivas mais eficazes e ampliem a consciência do seu processo de trabalho, assim como do desgaste decorrente deste, adquirindo conhecimento científico para prevenção dos agravos à saúde. Aos gestores dos serviços públicos e/ou privados, cabe a fiscalização e o investimento nos ambientes de trabalho, nos equipamentos de proteção individual e do ato de notificação dos agravos, tornando o ambiente mais seguro e minimizando os riscos de acidentes laborais.

\section{Referências}

Brasil, Ministério da Saúde, (2007). Doenças Relacionadas Ao Trabalho.

Brasil, Ministério da Saúde, (2017). Boletim Epidemiológico.

Brasil, Ministério da Saúde, (2020). Portaria nº 264, de 17 de fevereiro de 2020. Altera a Portaria de Consolidação nº 4/GM/MS, de 28 de setembro de 2017, Lista Nacional de Notificação Compulsória de doenças, agravos e eventos de saúde pública. Diário Oficial da União.

Brasil, Ministério do Trabalho e Emprego, (1994). Norma Regulamentadora 9: programa de prevenção de riscos ambientais. Portaria SSST.

Brasil. (2017). Lei $\mathrm{n}^{\circ} 13.467$, de 13 de julho.

Brasil. Ministério do Trabalho e Emprego, (2005). Portaria n 485, de 11 de novembro. Aprova a norma regulamentadora n 32 (Seg urança e saúde no trabalho em estabelecimentos de saúde). Diário Oficial da República Federativa do Brasil.

Brasil. Ministério do Trabalho e Emprego, (2010). Norma Regulamentadora - NR 5: comissão interna de prevenção de acidentes.

Carvalho, T.S., \& Luz, R. A. (2018) Acidentes Biológicos com Profissionais da Área da Saúde no Brasil: Uma revisão de literatura. Arq Méd. Doi: https://doi.org/10.26432/1809-3019.2018.63.1.31

Cavalcante, C. A. A., Cossi, M.S., Oliveira Costa, R. R., Medeiros, S., \& Menezes, R. M. P. (2015). Análise crítica dos acidentes de trabalho no Brasil. RAS. https://doi.org/10.13037/ras.vol13n44.2681

Conselho nacional de saúde, (2020). recomendação $n^{\circ}$ 033, de 05 de maio.

Dias, E. C., \& Hoefel, M. G. (2005). O Desafio de Implementar as Ações de Saúde do Trabalhador no SUS: a estratégia da RENAST. Ciênc Saúde Coletiva. https://www.scielosp.org/article/csc/2005.v10n4/817-827/

Empresa Brasileira de Serviços Hospitalares (EBSERH), (2018). 
Research, Society and Development, v. 10, n. 6, e8010615403, 2021

(CC BY 4.0) | ISSN 2525-3409 | DOI: http://dx.doi.org/10.33448/rsd-v10i6.15403

Fernandes, C., \& Pereira, A. (2016). Exposição a Fatores de Risco Psicossocial em Contexto de Trabalho: Revisão Sistemática. Rev Saúde Pública. https://doi.org/10.1590/S1518-8787.2016050006129

Galdino, A., Santana, V. S., \& Ferrite, S. (2012). Os Centros de Referência em Saúde do Trabalhador e a Notificação de Acidentes de Trabalho no Brasil. Cad. Saúde Pub. https://www.scielosp.org/article/csp/2012.v28n1/145-159/

Gomez, C. M., Vasconcellos, L. C. F., \& Machado, J. M. H. (2018). Saúde do trabalhador: Aspectos históricos, avanços e desafios no Sistema Único de Saúde. Ciênc Saúde Coletiva. https://doi.org/10.1590/1413-81232018236.04922018

Hämäläinen, P. Takala, J., \& Kiat, T. B. (2017). Global estimates of occupational accidents and work-related illnesses. Singapore: Workplace Safety and Health Institute.

Jacques, M. G., \& Jacques, C. C. (2009). Acidentes de Trabalho e Implicações Psicossociais: uma discussão introdutória. Pequi. Prát. Psicossociais. https://pesquisa.bvsalud.org/brasil/resource/pt/lil-600377

Meireles, N. M., \& Oliveira Pinto, F. A. (2016). Conscientização do trabalhador quanto à importância do uso do EPI na aerosolda em Macaé. Interdiscip. Scient.J. http://revista.srvroot.com/linkscienceplace/index.php/linkscienceplace/article/viewFile/221/133

Ministério da Saúde, Secretaria de Vigilância em Saúde, Departamento de Saúde Ambiental, do Trabalhador e Vigilância das Emergências em Saúde Pública, (2019). nota informativa no 94/2019-DSASTE/SVS/MS, Brasil.

Possebom, G., \& Alonço, A.S. (2018). Panorama dos acidentes de trabalho no Brasil. Nucleus. http://nucleus.feituverava.com.br/index.php/nucleus/article/view/2691

Santana, L.L., Sarquis, L.M.M., \& Miranda, F.M.A. (2020). Psychosocial risks and the health of health workers: reflections on brazilian labor reform. Rev Bras Enferm. http://dx.doi.org/10.1590/0034-7167-2019-0092

Secretaria de Previdência, (2020). Saúde e segurança: Brasil intensifica debates sobre saúde do trabalhador.

Sistema de Notificação de Agravos de Notificação. Ministério da saúde, Brasil (2016). Portaria nº 204, de 17 de fevereiro. 